\title{
Design Considerations for Aural Vital Signs Using PZT Piezoelectric Ceramics Sensor Based on the Computerization Method
}

\author{
Suranan Noimanee ${ }^{1, *}$, Tawee Tunkasiri ${ }^{2}$, Kingkeo Siriwitayakorn ${ }^{2}$ and Jerapong Tantrakoon ${ }^{2}$ \\ 1 Department of Computer Engineering, Chiang Mai University, 239 Huay Kaew Road, Chiang Mai \\ 50200, Thailand. Tel: +66 53 942018, Fax: +66 53942072
}

2 Department of Physics, Chiang Mai University, 239 Huay Kaew Road, Chiang Mai 50200, Thailand. Tel: +66 53 943376, Fax: +66 53892271

* Author to whom correspondence should be addressed. Email: suranan@chiangmai.ac.th Received: 23 July 2007 / Accepted: 3 December 2007 / Published: 11 December 2007

\begin{abstract}
The purpose was to illustrate how system developed for measurement of the aural vital signs such as patient's heart and lung sounds in the hospital. For heart sounds measurement must operate the frequency response between $20-800 \mathrm{~Hz}$, and lung sounds measurement must operate the frequency response between $160-4,000 \mathrm{~Hz}$. The method was designed PZT piezoelectric ceramics for both frequency response in the same PZT sensor. It converts a signal from aural vital sign form to voltage signal. The signal is suitably amplified and re-filtered in band pass frequency band. It is converted to digital signal by an analog to digital conversion circuitry developed for the purpose. The results were that all signals can fed to personal computer through the sound card port. With the supporting software for drawing of graphic on the screen, the signal for a specific duration is accessed and stored in the computer's memory in term of each patient's data. In conclusion, the data of each patient call dot pcg (.pcg) for drawing graph and dot wave (.wave) for sound listening or automatic sending via electronic mail to the physician for later analysis of interpreting the sounds on the basis of their time domain and frequency domain representation to diagnose heart disorders.
\end{abstract}

Keywords: aural vital signs, heart and lung sounds, PZT piezoelectric ceramics sensor, dot pcg, dot wave, interpreting the sounds 


\section{Introduction}

A stethoscope is one of the simplest basic diagnostic tools of a physician for assessing the health of a human patients. Although not everyone agrees as to what extent a stethoscope should be used, the aural vital signs sounds (such as heart and lung sounds) radiated by diseased heart and lungs are regarded by most clinicians as information of important diagnostic value. While even the most accurate sound analysis and display system alone is not sufficient for a successful diagnosis of diseases, a stethoscope can be a major component of a diagnostic system. The automatic signal process usually consists of acquiring signals originating from a source through PZT piezoelectric ceramic sensors, and then classifying these signals based upon some prior technology. The prior technology may include probabilities of occurrence of particular classes of signals, features of signals, or relations among the features. The classification decision rule can be implemented as a statistical classifier, a decision tree, a neural network or using some other classification approach. In this paper we present a methodology for model based feature selection and fusion for single-sensor detection, and show the results of the application of this methodology to heart and lung sounds display. Firstly, we describe our design of a system. Then we compare the resulting system with both a single-sensor system and with a system that selects features using an PZT piezoelectric ceramics sensor based feature selection method described. We begin with a brief overview of background of sensor which measure aural vital signs sound (second 2). In section 3 we describe the fundamental interpretation of aural vital signs are consist heart and lung sounds. Then, in section 4 we briefly describe our methodology and an Design Considerations for Aural Vital Signs Using PZT Piezoelectric Ceramics Sensor Based on the Computerization Method. By the way, we describe the steps of our methodology as applied to the design of an DCABSPZTC for this specific scenario. In section 5 we show the performance results of our system and compare them to a single-sensor system and to a single-sensor system that uses an entropy based measure for feature selection. Finally, in Section 6 we present our conclusions and directions for future research.

\section{PZT Piezoelectric Ceramics:}

PZT piezoelectric ceramics sensor offers a wide selection of piezoelectric ceramics are tailor made for diverse applications.

a). The material that exhibit a significant and useful piezoelectric effect fall into three main groups, such as:

i. Natural (quartz, rochelle salt)

ii. Synthetic (lithium sulfate, ammonium dihydrogen phosphate) crystals.

iii. Polarized ferroelectric ceramics (barium titanate, etc.)

b). The piezoelectric effect can made to respond to mechanical deformation of material in many different modes, such as:

i. Thickness expansion

ii. Transverse expansion

iii. Thickness shear

iv. Face shear. 
We illustrate the main characteristics of piezoelectric motion-to-voltage transducer by considering only one common mode of deformation, thickness expansion.

a. Two main families of constants, the $g$ constant and the $d$ constants, are considered.

i. Barium titanate thickness-expansion device, the pertinent $g$ constant is $g_{33}$. Which is defined as

$$
g_{33} \cong \frac{\text { field produced in direction } 3}{\text { stress applied in direction } 3}=\frac{e_{o} / t}{f_{i}}
$$

The main characteristics of piezoelectric motion-to-voltage transducer by considering only one common mode of deformation, thickness expansion. For this mode the physical arrangement is shows in figure $1 \mathrm{~b}$. Various double-subscripted physical constants numerically describe the phenomena occurring. The convention is that the first subscript refers to the direction of the electrical effect and the second to that the mechanical effect, by using the axis-numbering system of figure 1a.

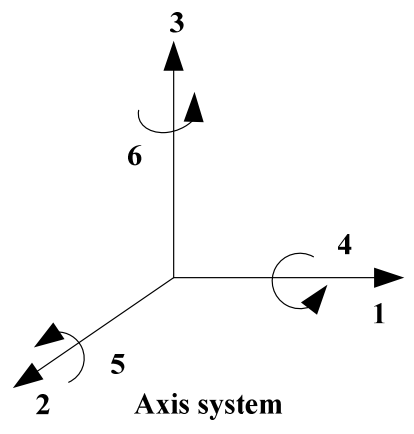

(a)

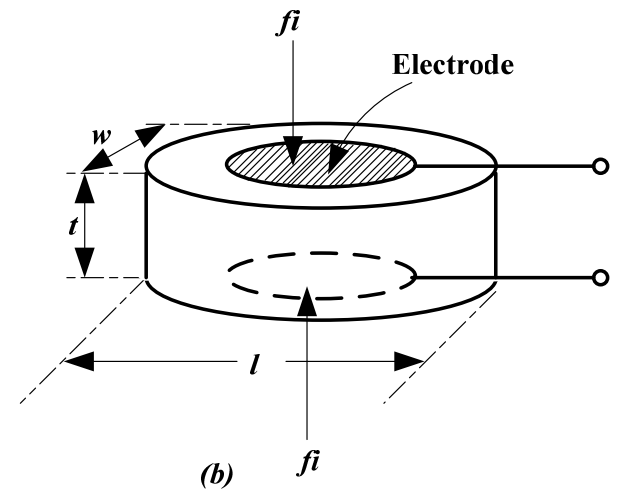

(b)

Figure 1. PZT Piezoelectric ceramics transducer.

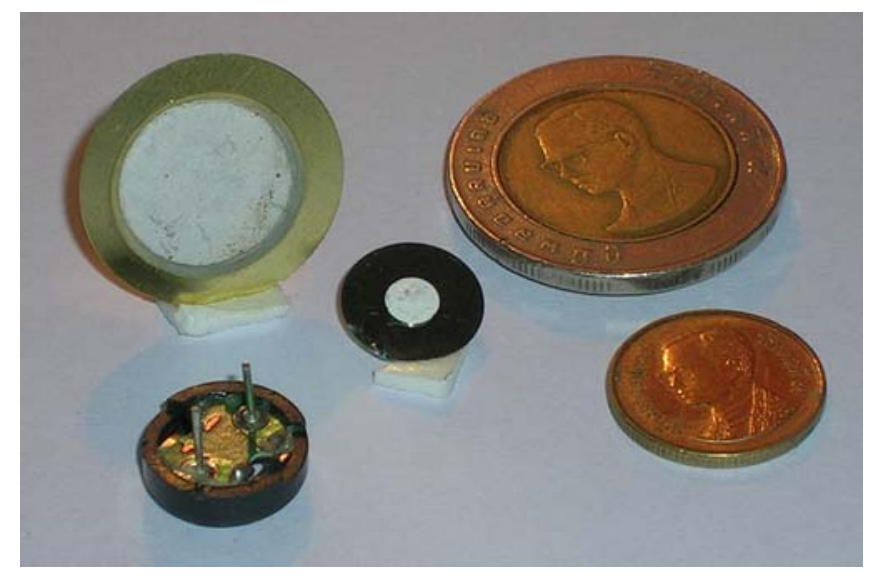

Figure 2. PZT Piezoelectric ceramics compared with Thai baht cone.

ii. The large voltage output for small stress typical of piezoelectric devices now for relate applied force to generated charge, the $d$ constants can be defined as 


$$
d_{33} \cong \frac{\text { charge generated in direction } 3}{\text { force applied in direction } 3}=\frac{Q}{f_{i}}
$$

iii. As for $\mathrm{d} 33$ can be calculated from the value of $\mathrm{g} 33$ if the dielectric constant $\varepsilon$ of the material is known, since

$$
\begin{aligned}
& C=\frac{\varepsilon w l}{t} \\
& g_{33} \cong \frac{\text { field }}{\text { stress }}=\frac{e_{o} w l}{t f_{i}}=\frac{e_{o} C}{\varepsilon f_{i}}=\frac{Q}{\varepsilon f_{i}}=\frac{d_{33}}{\varepsilon} \\
& d_{33}=\varepsilon g_{33}
\end{aligned}
$$

The dielectric constant of quartz is about $4.06 \times 10^{-11} \mathrm{~F} / \mathrm{m}$ while for barium titanate it is $1,250 \mathrm{x}$ $10^{-11}$. For quartz, then,

$$
d_{11}=\operatorname{cg}_{11}=\left(4.06 \times 10^{-11}\right)\left(50 \times 10^{-3}\right)=2.03 \mathrm{pC} / \mathrm{N}
$$

For transducer alone, if a static deflection $x_{i}$ is applied and maintained, a transducer terminal voltage will be developed but the charge will slowly leak off through the leakage resistance to the transducer. Since $R_{\text {leak }}$ is generally very large (in order of $10^{11} \Omega$ ), this decay would be very slow, perhaps allowing at least a quasi-static response. However when the external voltage-measuring device of low input impedance is connected to the transducer, the charge off very rapidly, preventing the measurement of static displacements. Even relatively high-impedance amplifiers generally do not allow static measurements. Some researcher using quartz transducers (very high leakage resistance) and electrometer input amplifiers (very high input impedance) achieve total resistance of $10^{14} \Omega$, which gives a sufficiently slow leakage to allow static measurements.

iv. We consider Figure 3. the charge generated by the crystal can be expressed as

$$
q=K_{q} x_{i}
$$

where

$$
\begin{aligned}
& K_{q} \cong C \mathrm{~cm} \\
& x_{i} \cong \text { deflection }
\end{aligned}
$$

The resistances and capacitances of Figure $3 \mathrm{~b}$ can be combined as in $3 \mathrm{c}$. we also convert the charge generator to a more familiar current generator according to 


$$
i_{c r}=\frac{d q}{d t}=K_{q}\left(\frac{d x_{i}}{d t}\right)
$$

We may then write

$$
\begin{aligned}
& i_{c r}=i_{C}+i_{R} \\
& e_{o}=e_{C}=\frac{\int i_{C} d t}{C}=\frac{\int\left(i_{c r}-i_{R}\right) d t}{C} \\
& C\left(\frac{d e_{o}}{d t}\right)=i_{c r}-i_{R}=K_{q}\left(\frac{d x_{i}}{d t}\right)-\frac{e_{o}}{R} \\
& \frac{e_{o}}{x_{i}}(D)=\frac{K \tau D}{\tau D+1}
\end{aligned}
$$
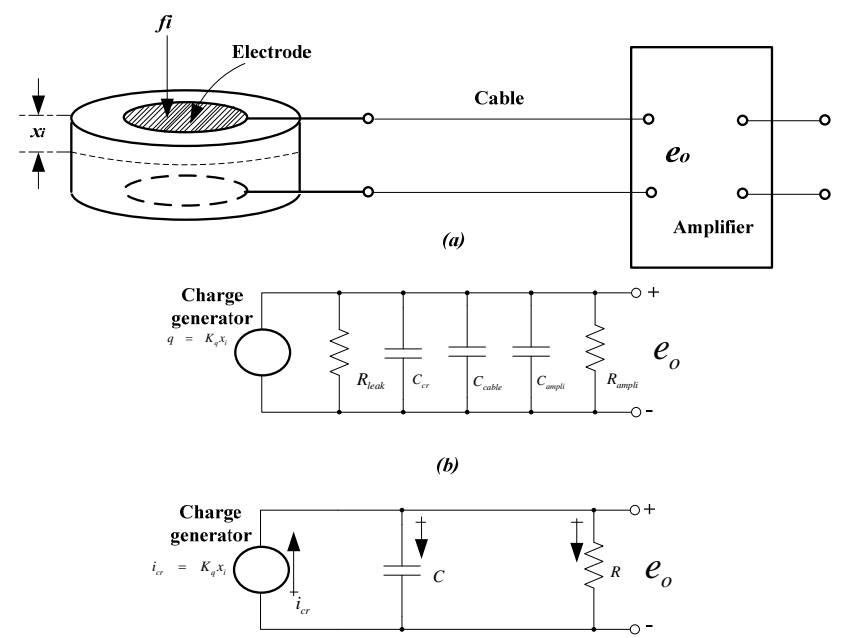

(c)

Figure 3. Equivalent circuit for piezoelectric ceramic.

where

$$
\begin{aligned}
& K \cong \text { sensitivity } \cong \frac{K_{q}}{C}, \mathrm{~V} / \mathrm{cm} \\
& \tau \cong \text { time cons } \tan t \cong R C
\end{aligned}
$$

We see that, just as in the capacitance pickup of Figure 4, the steady-state response to a constant $x_{i}$ is zero.

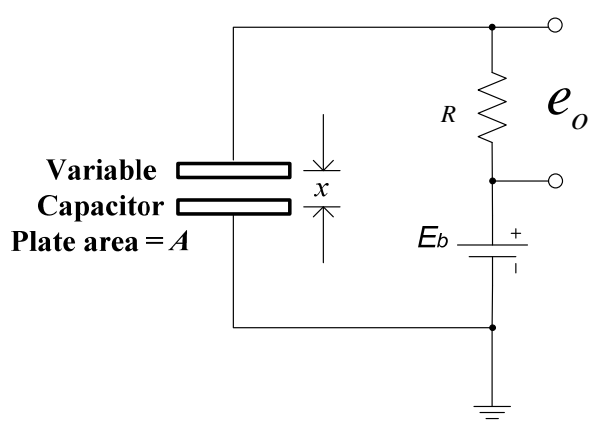

Figure 4. Capacitive transducer. 
Thus we can not measure static displacements. For a flat amplitude response within, say, $5 \%$, the frequency must exceed $\omega_{1}$, where

$$
\begin{aligned}
& 0.95^{2}=\frac{\left(\omega_{1} \tau\right)^{2}}{\left(\omega_{1} \tau\right)^{2}+1} \\
& \omega_{1}=\frac{3.04}{\tau}
\end{aligned}
$$

$\mathrm{v}$. The response of PZT piezoelectric ceramic is further illuminated by considering the displacement input of Figure 5. The differential equation is

$$
(\tau D+1) \mathrm{e}_{\mathrm{o}}=(K \tau D) x_{i}
$$

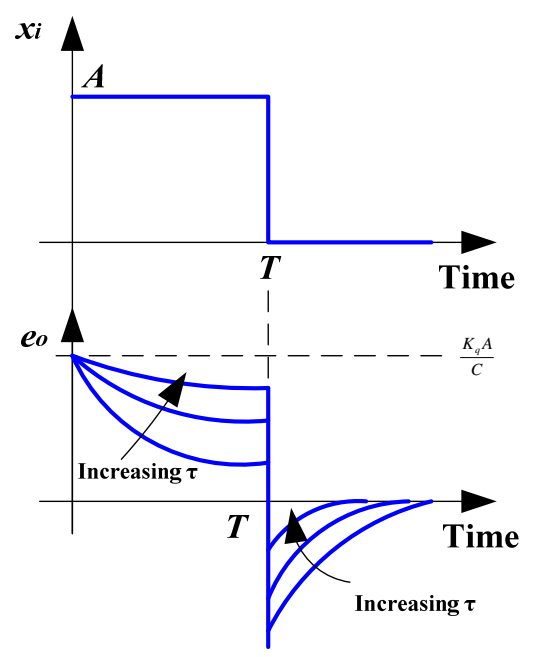

Figure 5. Pulse response of piezoelectric ceramic.

Anyway Figure 5. shows the complete process for three different values of $\tau$. It is clear that a large $\tau$ is desirable for faithful reproduction of $x_{i}$. if the decay and "undershoot" at $t=T$ are to be kept within, say $5 \%$ of the true value, $\tau$ must be at least $20 \mathrm{~T}$. if an increase of $\tau$ is required in a specific application, it may be achieved by increasing either or both $\mathrm{R}$ and $\mathrm{C}$. An increase in $\mathrm{C}$ is easily obtained by connecting an external shunt capacitor across the piezoelectric ceramic terminals. Since shunt capacitors add directly. The price paid for this increase in $\tau$ is a loss of sensitivity according to $\mathrm{K}$ $=\mathrm{K}_{\mathrm{q}} / \mathrm{C}$. Often this may be tolerated because of the initial high sensitivity of piezoelectric devices. An increase in $\mathrm{R}$ generally requires an amplifier of greater input resistance.

b. PZT piezoelectric ceramics materials compare favorably with the best materials internationally available today.

i. Material is the standard material for actuators and suitable for low-power ultrasonic transducers and low-frequency sound transducers. PZT piezoelectric ceramics is a modified lead zirconate-lead titanate piezoelectric ceramics material with extremely high permittivity and coupling factors, a high charge constant, and a Curie temperature of around $185^{\circ} \mathrm{C}$. This material is suitable for hydrophones, transducers in medical diagnostics and PZT translators 
c. Phenomenological equations that describe the behavior of a piezoelectric material subjected to a small stress and/or a weak electric field may be derived from the thermodynamic potentials.

i. The piezoelectric response consists of the sum of intrinsic and extrinsic effects. The intrinsic effect results from the response of a single domain crystal under the application of an electric field.

ii. The extrinsic effect results from the elastic deformation caused by the motions of the non $-180^{\circ}$ domain walls. Under the application of an electric field, the domain wall moves to minimize the domain energy. Contributions from the electrostatic and domain wall energies counteract each other and the underlying domain structure (size, shape, and population) changes. In this process some of the domains engulf other domains or change shape, all of which changes the net strain and polarization. One set of such equations is:

$$
\begin{aligned}
& D_{i}=\varepsilon_{i j}^{T} E_{j}+d_{i m} T_{m} \\
& S_{k}=d_{j k} E_{j}+s_{k m}^{E} T_{m}
\end{aligned}
$$

where $E, D, T$, and $S$ are the electric field, electric displacement, stress, and strain, respectively. The constants $\mathrm{s}^{\mathrm{E}}, \mathrm{e}^{\mathrm{T}}, d$ are the elastic compliance at constant electric field, the dielectric permittivity under constant stress, and the piezoelectric coefficient, respectively. The phenomenological equations shown above can be uncoupled thereby allowing the strain and dielectric displacement to be monitored as a function of electric field, which facilitates the determination of the piezoelectric coefficients.

\section{The fundamental interpretation of aural vital signs are consist heart and lung sounds.}

\section{a). Heart sounds}

i. $\mathbf{S}_{1}$ : First heart sound is initiated by the onset of ventricular systole. It is the longest and loudest of the four sounds. $S_{1}$ is heard best over the apical region of the heart. It is produced by the oscillation of blood in the ventricular chambers and vibration of the ventricular free wall. At the onset of systole, ventricular pressure begins to increase and forces the blood within the ventricles toward the atria through the mitral and tricuspid valves. As these one way valves close, blood and the ventricular wall vibrates producing the $S_{1}$ sound. Therefore, $S_{1}$ sound is expected soon after the onset of the ventricular contraction comparison with the electrical activity generated by the heart. The recorded tracing is called an $\mathrm{Q}, \mathrm{R}$, and $\mathrm{S}$ waves that represent the ventricular activity of the heart. Heart now is in the iso-volumetric contraction phase of the cardiac cycle.

ii. $S_{2}$ : The second heart sound is generated the end of the ejection of the blood from the ventricles by the closure of the semi-lunar valves, i.e. aortic and pulmonic valves. At this point, blood from the ventricle is pumped into the arteries, arterial pressure exceeded the ventricular pressure, hence the one way valves are closing as the pressure is reversed. These sounds have higher frequency components and the aortic valve sound is louder than the pulmonic valve sound. 
iii. $\mathbf{S}_{3}$ : This heart sound is heard in young children and patients with left ventricular failure and believed to be resulted by the deceleration of blood entering the ventricle. In enlarged hearts, ventricular walls are stretched beyond normal ranges and are under tension allowing vibrations.

iv. $\mathbf{S}_{4}$ : Is the weakest on of all the heart sounds and heard occasionally. It is not related to a pathological condition and believed to be due to the oscillation of blood in the atria and atrial walls. It occurs immediately after the P-wave in the Electrocardiogram (ECG). The basic points for heart sounds quantitative display and analysis are according to the model for heart and lung sounds generation and propagation, the mean amplitude of white noise, mainly intra cardiac and cardiothoracic, was abstracted and used as the waveform baseline, thus rendering the amplitude of heart and lung sounds components relative to the baseline.

v. Measuring the duration for each component of the heart sounds $\left(S_{1}\right)$, second heart sounds $\left(\mathrm{S}_{2}\right)$, etc. Based on the data obtained from the above measurements, indicators generated for heart and lung sounds diagnosis were offered to the classification program. There is no criterion for amplitude measurement of heart sounds in cardiology, so quantitative analysis must be used to established a standard. And used a sampling rate of $32 \mathrm{KHz}$ with 10 bits per sample. Amplitude resolution is represented by the ratio of the amplitude of the heart and lung sounds component to the baseline. If a peak amplitude of a heart and lunge sounds sample are three times or more greater than that of the baseline, the peak is easily recognized by both the human eyes and computer. Therefore, the amplitude resolution of this system can be evaluated as being the percentage of the number of subjects whose heart or lunge sound peak values are above this criterion. Because the duration of each sound component can be easily determined by the sampling rate used, we choose the duration of the cardiac cycle measurement as the parameter to be evaluated. For every test of each subject, the cardiac cycle data were measured and recorded with heart sounds quantitative analysis by computer software and conventional methods.

vi. Exercise level was determined by initial evaluation of the subject's cardiovascular status. There are many protocols for increasing the exercise workload of the aural vital signs, but they all share the common principle of progressively increasing the grade and/or speed of the exercise test as shown in Figure 6.

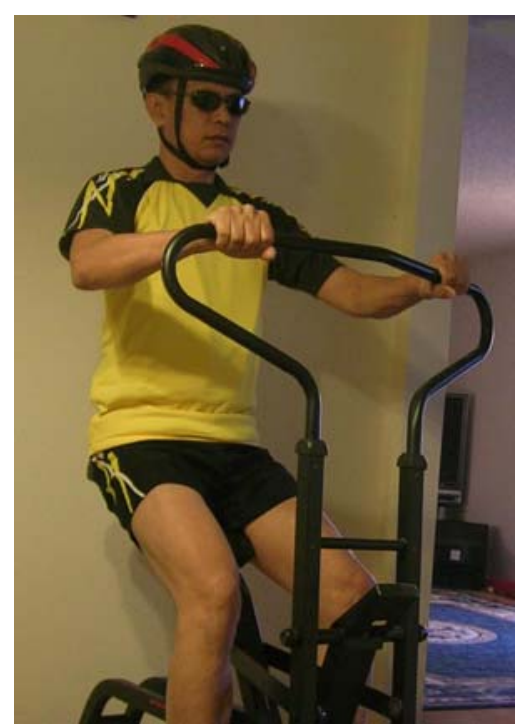

Figure 6. The exercise test before measuring heart and lung sounds. 
vii. The end point of the exercise test is usually a limitation by symptoms, or the achievement of a certain target heart rate (80-95\%) of the maximal achievable heart rate. The proposed aural sound measurement using piezoelectric ceramic method was applied to the data collected from the health screening of elementary Chiang Mai university students. The medical interpretation method which utilizes a quarter of the total data for evaluation was used for measuring the performance. The correct detection rate of the systolic murmur was $78.6 \%$ with the false alarm rate of $84.5 \%$. Typical examples of time frequency distribution of aural sound data such as heart and lunge sound for normal and innocent murmur were shown in Figure 7. It is clearly seen the time frequency power distribution is narrower for the innocent murmur compared to the abnormal murmur. The result of classification utilizing the diagnosis heart diseases over the time frequency power distribution was shown in the table 1 .

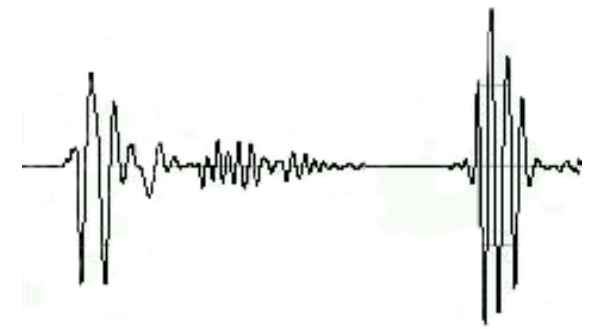

Figure 7. Normal and innocent murmur $(\mathrm{t}=1.528 \mathrm{~s})$.

Table 1. Compare with heart and lung sounds.

\begin{tabular}{|c|c|c|}
\cline { 2 - 3 } \multicolumn{1}{c|}{} & Sound & Frequency $(\mathbf{H z})$ \\
\hline \multirow{3}{*}{ Heart } & $\begin{array}{c}\text { Low pitched heart murmurs } \\
\text { High pitched heart murmurs } \\
\text { murmurs }\end{array}$ & $\begin{array}{c}400 \\
\text { Systolic and Diastolic }\end{array}$ \\
& $\begin{array}{c}\text { Presystolic murmurs } \\
\text { Pericardial rub }\end{array}$ & $120-660$ \\
\hline \multirow{3}{*}{ Lungs } & Rales & $140-660$ \\
& Amphonic breathing & $120-1,000$ \\
& Bronchial & $240-660$ \\
& & $240-1,000$ \\
\hline
\end{tabular}

\section{b). The lung sounds:}

i. In this section we show examples of lung sounds and common lung diseases in which these sounds are present. Lung sounds that can be heard through a stethoscope can be classified into two groups normal breathing sounds that occur when no respiratory problems exist and adventitious (abnormal) sounds when a problem exists. The normal breath sounds are both inspiratory and expiratory. They occur when the air moves in and out of the chest during a regular breathing cycle. The normal sounds are classified according to the character of the sound and the location where this sound is heard. The amplitude and frequency of these sounds can vary from location to location and from person to person. Abnormal sounds are those sounds that occur unexpectedly during a regular 
breathing cycle. They include normal sounds, when they appear at atypical locations, and adventitious sounds. For example, sounds of a bronchial or bronchovesicular nature are considered to be abnormal if they appear in locations where vesicular sounds should be heard. Considerable variability in the character of lung sounds among different individuals makes it sometimes difficult to be sure of the "abnormality" of a sound.
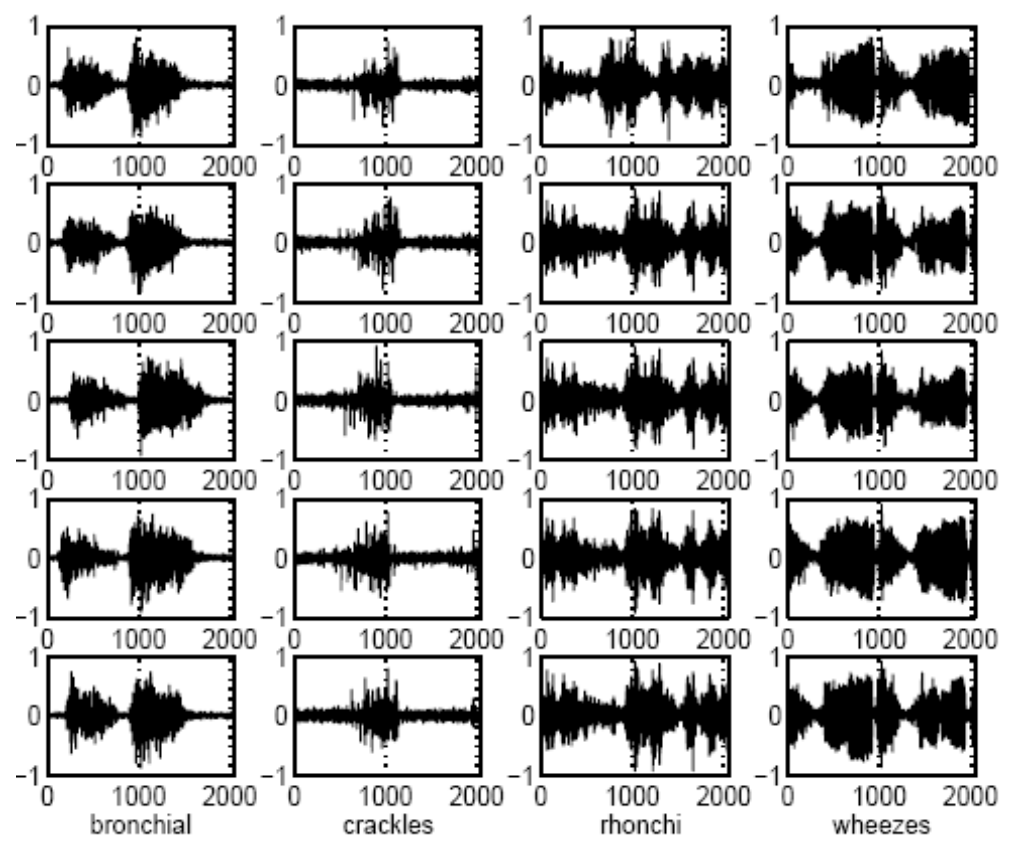

Figure 8. 5 signatures for each of the 4 classes of lung sounds in normalized amplitude vs time in $\mathrm{mS}$.

Figure 8 shows five examples of lung sound signatures for each of the four classes of lung sounds in type of lung sounds. Additionally, we created two test database one for PZT and one for other electronic stethoscope. They were generated by first picking specific signals, breathing cycles from the tape and then adding noise to the signals. For each class of signals we selected five signals (different than the signals selected for the reference databases) and then added zero-mean noise on eleven levels of standard deviation.

There are two major types of adventitious sounds: continuous and discontinuous. Among continuous adventitious sounds_the most well known are rhonchi, wheezes and stridor. Wheezes are continuous sounds, longer than $250 \mathrm{mS}$, high pitched, with dominant frequency of $400 \mathrm{~Hz}$ or more (a hissing sound). Rhonchi are also longer than $250 \mathrm{mS}$ in duration, low pitched, with dominant frequency of $200 \mathrm{~Hz}$ or less, (a snoring sound). Stridor is similar to wheezes. Both are high-pitched, but stridor can often be distinguished by its tendency to be accentuated in inspiration. The most common discontinuous adventitious sounds are crackles and squawks. Crack les are discrete, nonmusical sounds, usually shorter than $10 \mathrm{mS}$. They are a sequence of short interrupted sounds with a wide spectrum of frequencies between 120 and 2,000 Hz. The characteristics number and timing of crackles vary in different diseases. Fine crackles are characterized by the initial detection width of 0.9 $\mathrm{ms}$ and two cycle duration of $6 \mathrm{~ms}$, while these characteristics for coarse crackles are $1.25 \mathrm{~ms}$ and 9.5 ms, respectively. Squawks are a combination of wheezes and crackles, they may start with fine 
crackles and then sound like short inspiratory wheezes. The sounds of diseased lungs are regarded by most of clinicians as having a relatively high diagnostic value.

ii. The theoretical prediction of the speed of sound in two-phase system as described by Wallis was used to develop the following expression for the sound speed as a function of the volume fraction of air in the lungs - eg.22:

$$
C=\left[\left(v P_{A}+(1-v) p_{T}\right) \times\left(\frac{v}{p_{A} C_{A}^{2}}+\frac{1-v}{p_{T} C_{T}^{2}}\right)\right]^{-0.5}
$$

iii. $\mathrm{C}$ is the speed of sound in a two-phase system, $v$ is the fraction of air by volume; $\mathrm{V}_{\mathrm{A}}$ volume of air; $\mathrm{V}_{\mathrm{T}}=0.800 \mathrm{~L}$ volume of tissue; $\mathrm{p}_{\mathrm{A}}=1.293 \mathrm{~kg} / \mathrm{m}^{3}$ air density; $\mathrm{p}_{\mathrm{T}}=0.998 \mathrm{x} 103 \mathrm{~kg} / \mathrm{m}^{3}$ tissue density; $\mathrm{C}^{\mathrm{A}}=344 \mathrm{~m} / \mathrm{s}$ speed of sound in the air, $\mathrm{C}_{\mathrm{T}}=1460 \mathrm{~m} / \mathrm{s}$ speed of sound in the tissue. For the purpose of simplicity tissue density was assumed to be spatially constant and independent of orientation with the gravitational field.

$$
C=\frac{1}{\sqrt{v(1-v)}} \sqrt{\frac{p_{A}}{p_{T}} C_{A}}
$$

iv. Classification was done for each beat. Diagnosing heart diseases with a stethoscope is one fundamental methods because of its efficiency, simplicity and non-invasive property. Heart and lung sounds auscultation highly depends on the hearing ability, skill and experience of a cardiologist. Therefore, a computerized heart and lung sounds analysis is vital to assists the cardiologist. The heart and lung sounds need to be segmented into it's components before any automatic analysis can be applied. The main components of heart sounds are first heart sound $\left(\mathrm{S}_{1}\right)$ and second heart sound $\left(\mathrm{S}_{2}\right)$. $\mathrm{S}_{1}$ occurs during ventricular systole and it contributes to the 'lub' of the 'luhdub' characteristic that can be heard from each heartbeat. It is caused by the closure of the mitral and tricuspid valves. Mean while, $\mathrm{S}_{2}$ occurs during ventricular diastole and it contributes to the 'dub'. It is caused by the closure of the aortic and pulmonary valves. $\mathrm{S}_{3}$ occurs just after $S_{2}$ and has relatively lower energy. $\mathrm{S}_{4}$ occurs just before the $S_{1}$ and has lower amplitude compared to the other heart sounds. The opening and closing of cardiac valves and the sounds they produce are mechanical events of the cardiac cycle. They are preceded by the electrical events of the cardiac cycle. Heart murmurs are noises associated with the damage of valves and improper closure of valves. The following is the relationship between the Phonocardiogram (PCG) and ECG in time domain. The $\mathrm{S}_{1}$ occurs $0.04 \mathrm{~s}-0.06 \mathrm{~s}$ after the onset of the QRS complex, the $\mathrm{S}_{2}$ occurs towards the end of the' $\mathrm{T}$ wave, and the fourth heart sound $\mathrm{S}_{4}$ occurs after the $\mathrm{P}$ wave.

v. Some research has been done to segment heart sound signals. We wanted to develop a detection algorithm for $S_{1}$ and $S_{2}$ based on frequency domain of PCG evaluated by computerized method. Therefore, a computerized heart sound analysis is vital to assists the cardiologist. The heart sounds need to be segmented into it's components before any automatic analysis can be applied. Heart sound consists of 4 components which are $\mathrm{S}_{1}, \mathrm{~S}_{2}, \mathrm{~S}_{3}$ and $\mathrm{S}_{4}$ but $\mathrm{S}_{4}$ sound is difficult to hearing. Again, the main components of heart sound are first heart sound $\left(\mathrm{S}_{1}\right)$ and second heart sound $\left(\mathrm{S}_{2}\right)$.

In Figure 9 shows where $\mathrm{S}_{1}$ is estimated by using the onset of the R wave in ECG and the beginning of $S_{2}$ by using the carotid pulse. As reference where $S_{1}$ is estimated by using the onset of the 
$\mathrm{R}$ wave in ECG and the beginning of $\mathrm{S}_{2}$ by using the carotid pulse. This method fails to perform properly due to the timing between electrical and mechanical activities that vary to a larger extent. The nature of these two signals in time domain may not be exactly constant but a good deal of agreement exists between them in spectral components. But, the direct application of the spectrum analysis is not suitable since the spectrum can not detect the temporal variation in the heart sound and electrocardiogram. As, heart sound and ECG is time varying signal, Instantaneous Energy is applied to characterize the temporal behaviors of those signals. The purpose of this study is to develop an algorithm to detect the occurrence of $\mathrm{S}_{1}$ and $\mathrm{S}_{2}$ and thus perform heart sound segmentation based on the instantaneous energy of electrocardiogram.

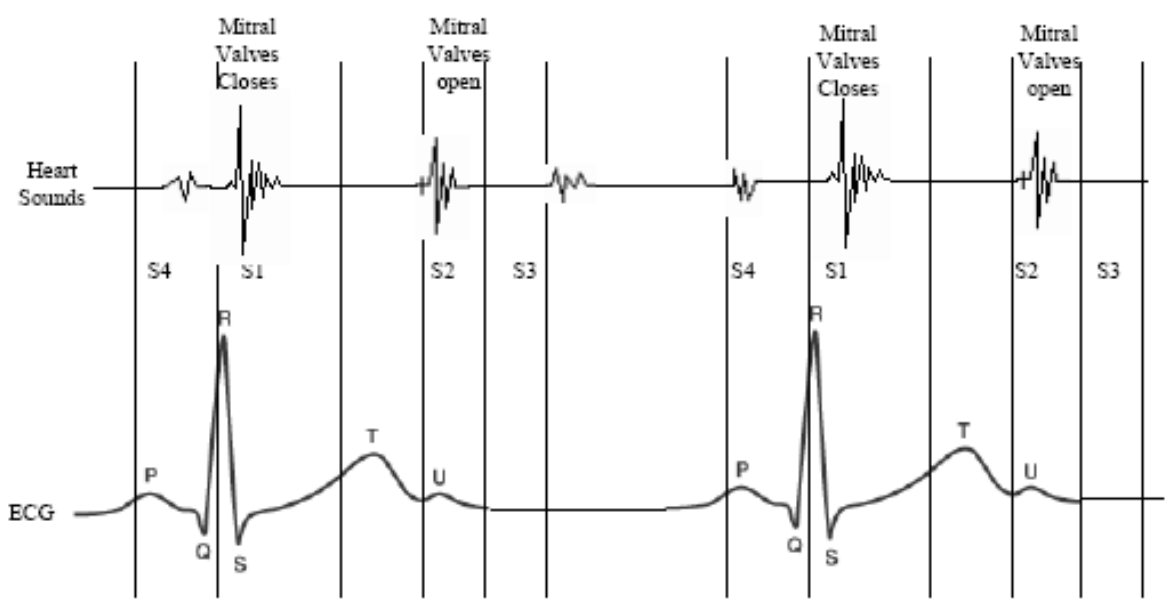

Figure 9. Comparison between ECG and PCG timing.

\section{Methods and Materials}

\subsection{System Overviews}

a. Our system, called the aural vital-signs measurement using PZT piezoelectric ceramics based on the computerization method system consists of four main processing blocks. Aural vital-signs preamplifier, filter path, analog to digital conversion path and software path. The system for measurement of human intestinal tract activity is designed for recording of one heart sound signal and one lungs sound signal measured relatively to one reference PZT piezoelectric ceramics. Block diagram of the measuring system is shown in Figure 10. Heart and lung sounds signal are sensed by a set of PZT piezoelectric ceramics is inserted in stethoscope (Figure 14) placed in commonly used positions in the abdominal region. Appropriate transducers on the chest monitor various sounds parameters. The data are processed in a microcontroller with a memory buffer and encrypted for transmission in bursts by means of a PCs interface, which is carried by the body chest. 


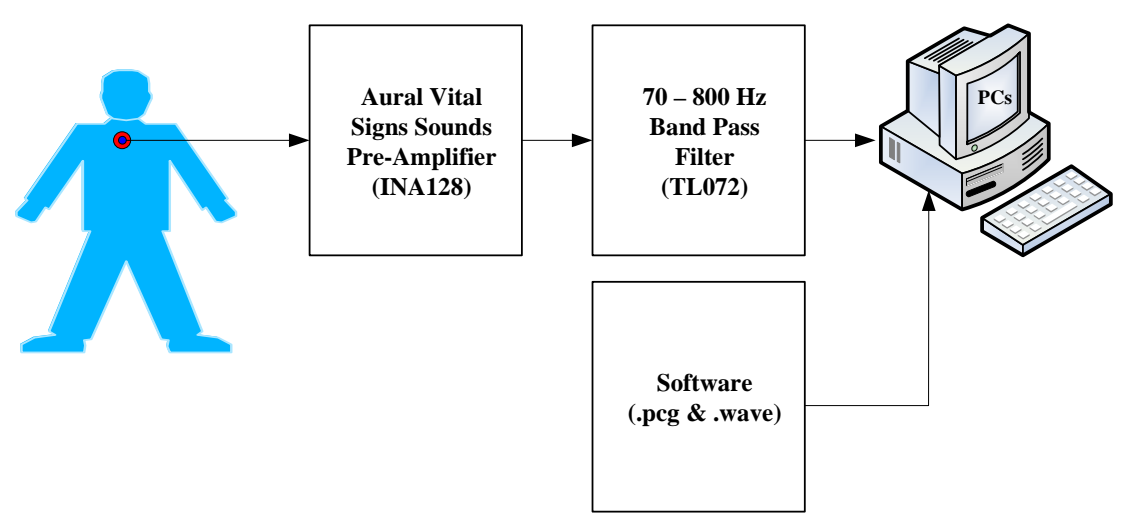

Figure 10. Block diagram of the system.

b. The data from PZT piezoelectric ceramics transducers are received and processed by a PIC family microcontroller connected to an appropriate port of a personal computer or PCs by Texas Instrument Semiconductor. Data buffering in local memory at the receiver is required to cater for different rates of communication in the system. The PCs is connected to the hardware set. Each general based PCs acts as a decentralized supervisory controller and has applications and local storage for the acquired data.

c. The real-time software recording system enables the types of transducers and the parameters of use to be chosen with the aid of graphical user interfaces displayed on screen of PC. These are dynamically created by using visual basic software and customized for the monitor type and individual person. Access to parameter value selection is more tightly controlled than reading of data.

d. The data from the monitors is continuously acquired and stored in temporary buffers. The real-time recording software acts as a supervisory control that continuously interacts with the data in these buffers. It formats the buffered data so that it can be displayed on request or when triggered by the system. Visual basic software is required to connect the data so it can be viewed in a PCs. The buffered data is passed periodically to a centralized database where records are analyzed and stored by a Database Management System.

e. Real-time systems are required to be resilient in the presence of errors. Transactions are logged and a "watch-dog" confirms that the system is working correctly. Data exceptions that may be caused by mouse actions in disturbing correct monitor operation need to be distinguished from technical communication errors. The software attempts to recover from these inadvertent data excursions.

f. System failures may require users to be informed about the reduction in quality of the information that is available. The function of the centralized records software system is to record, modify, analysis and retrieve patients information. At the centre is a database. This database has two sources of input: human data-entry of information and requests via hardware interface, and continuous updates from the real-time recording software temporary buffers, which store the data that monitor people's health. 


\section{Results}

The majority of patients had predominantly positive polarity of inspiratory crackles $(80 \%$ of patients). The majority of patients had predominantly positive polarity of exspiratory crackles (75\%). The frequency of inspiratory crackles tended to higher than frequency of expiratory crackles. Because of this $70-800 \mathrm{~Hz}$ frequency response of the PZT piezoelectric ceramics is shown in Figure 11. And Figure 14 as shown the hardware of interpreting system of aural-vital signs prototype which implementation at Chiang Mai University. We have PZT piezoelectric ceramics with a diameter 4.0 mm. shows in Figure 12. We successfully made the measurement of heart and lung sounds with tree main designs. First, the design was taken using PZT sized that expanded in range $6.0 \mathrm{~mm}$ for heart sound measurement and $4.0 \mathrm{~mm}$ in diameter for lung sounds measurement. Next, the PZT packet was inserted into stethoscope device. Finally, the device could measured and displayed three and four heart sounds on PC's screen as shown in Figure 15.

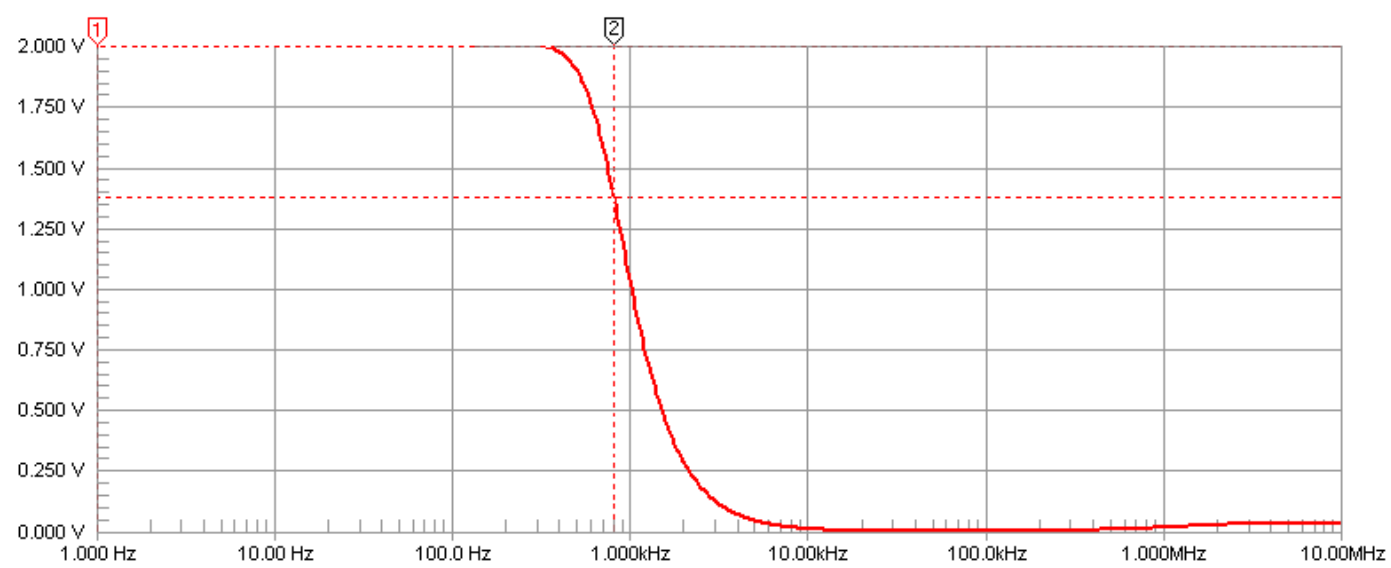

Figure 11. Frequency response of PZT.

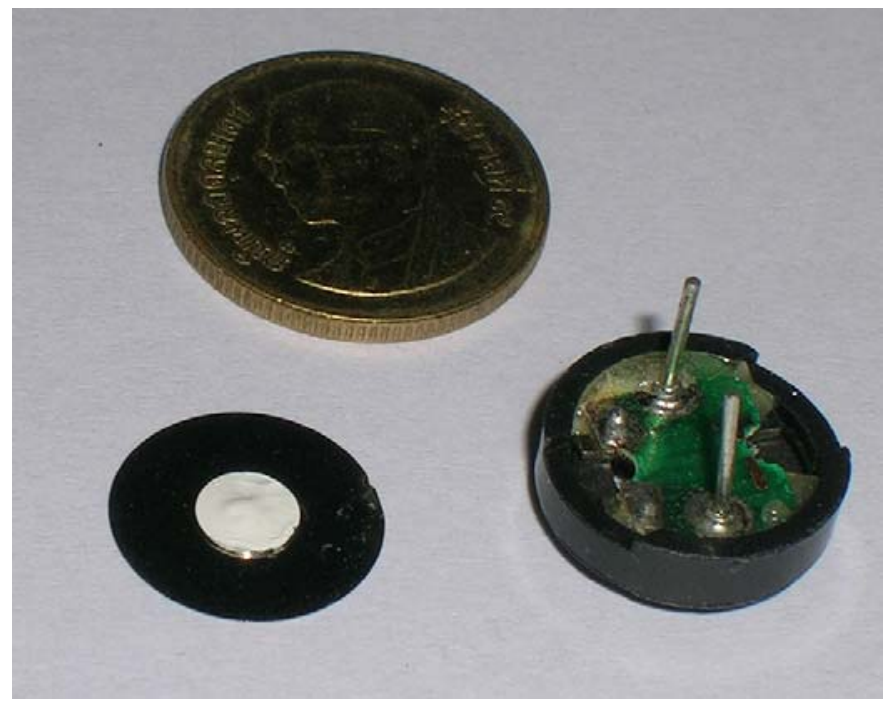

Figure 12. PZT piezoelectric ceramics with 4.0 m.m. diameter. 


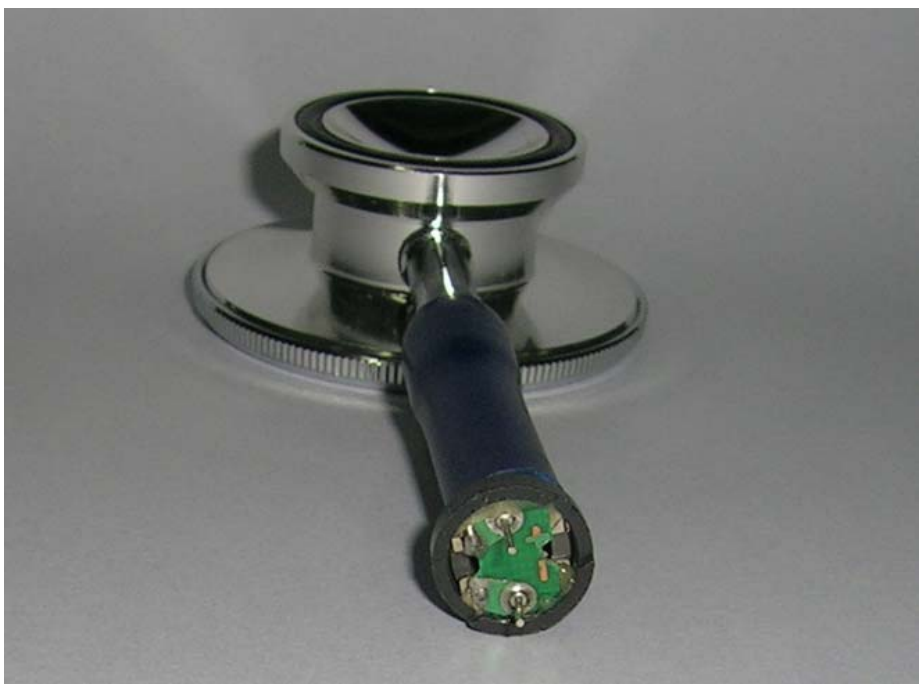

Figure 13. PZT is inserted in stethoscope.

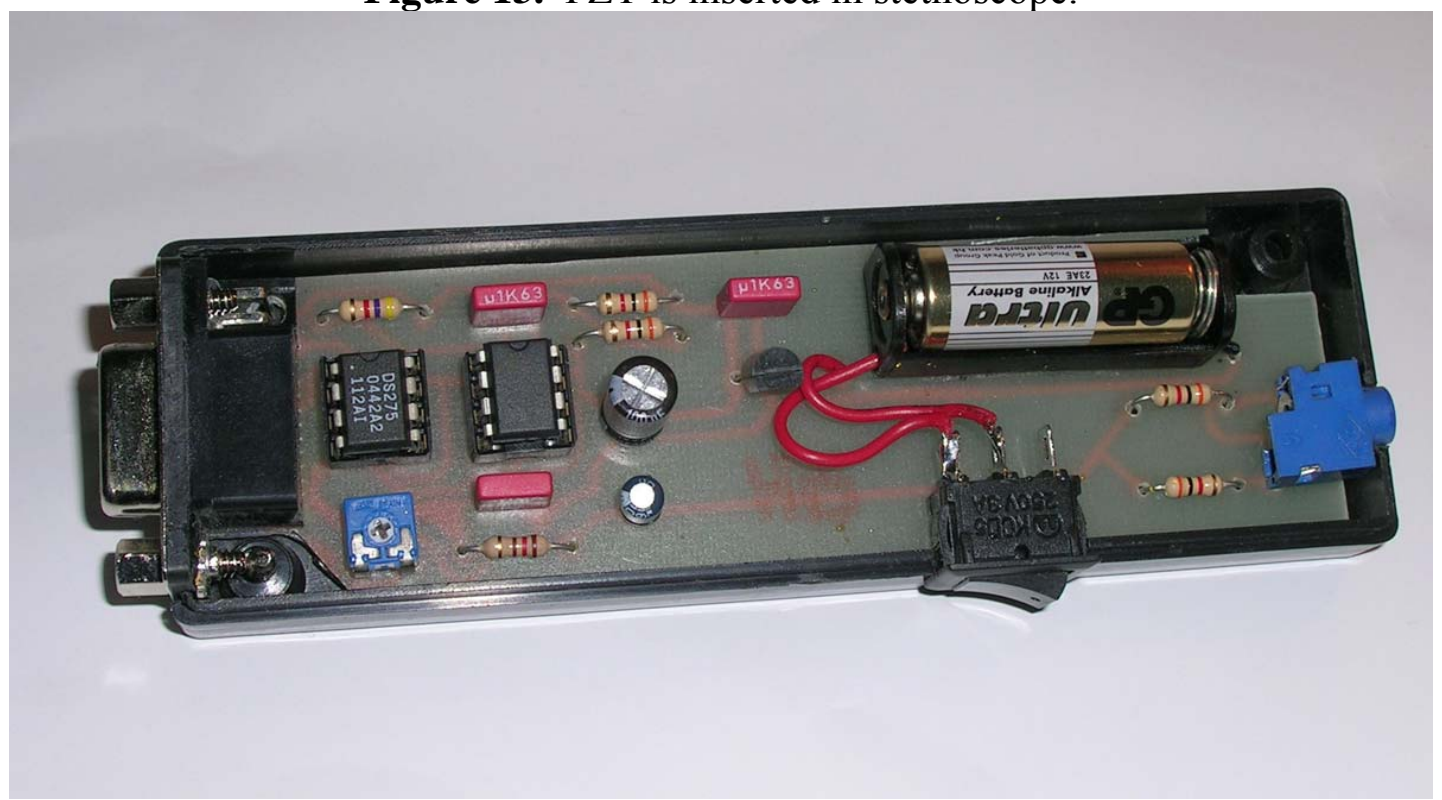

Figure 14. Prototype of aural vital signs system.

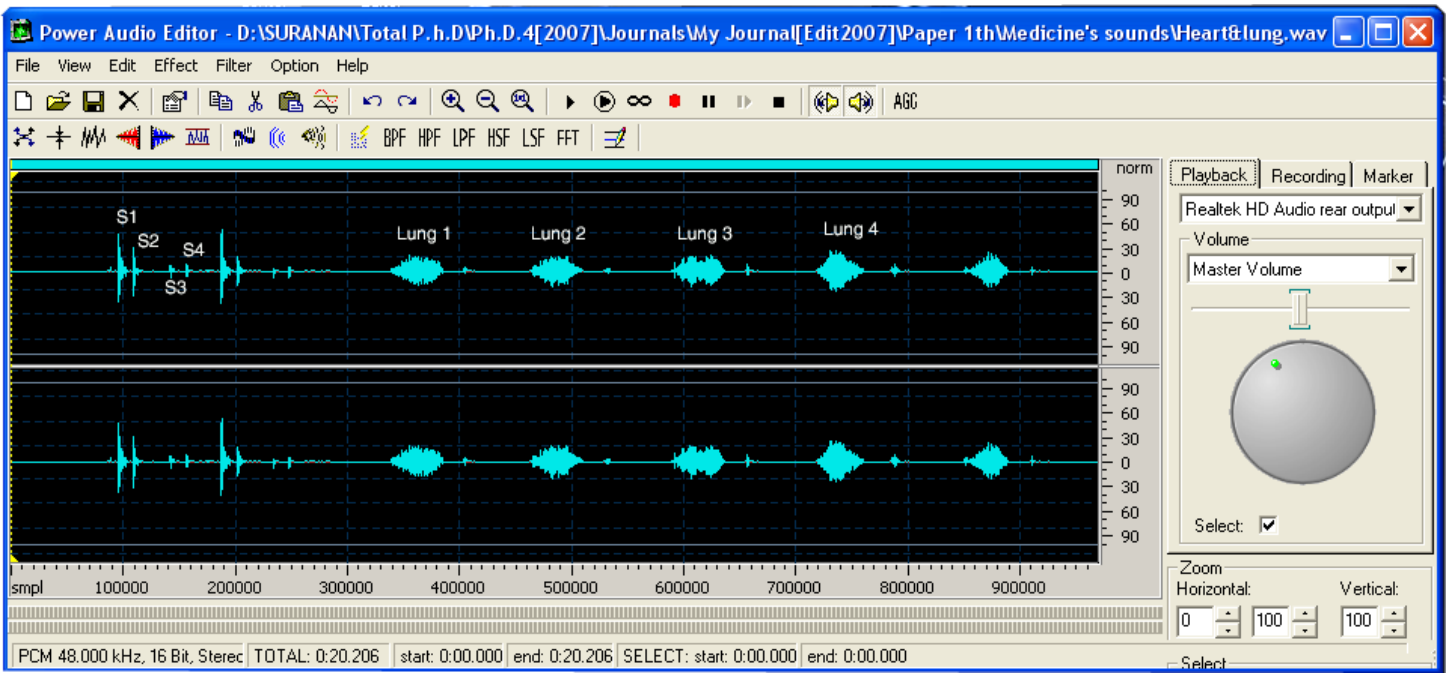

Figure 15. The heart and lung sounds output display. 
An example of the transit time analysis through right chest of the polyphonic sound with frequencies of 70 to $140 \mathrm{~Hz}$ is shown in Figure 16A, the sound recorded at the reference is shown on the top and the lung sound recorded is shown on the bottom. Using the time interval between corresponding peaks of two sounds to yield an approximation of transit time, the sound recorded from PC screen is delayed by $14.9 \mathrm{~ms}$ as compared to the reference.

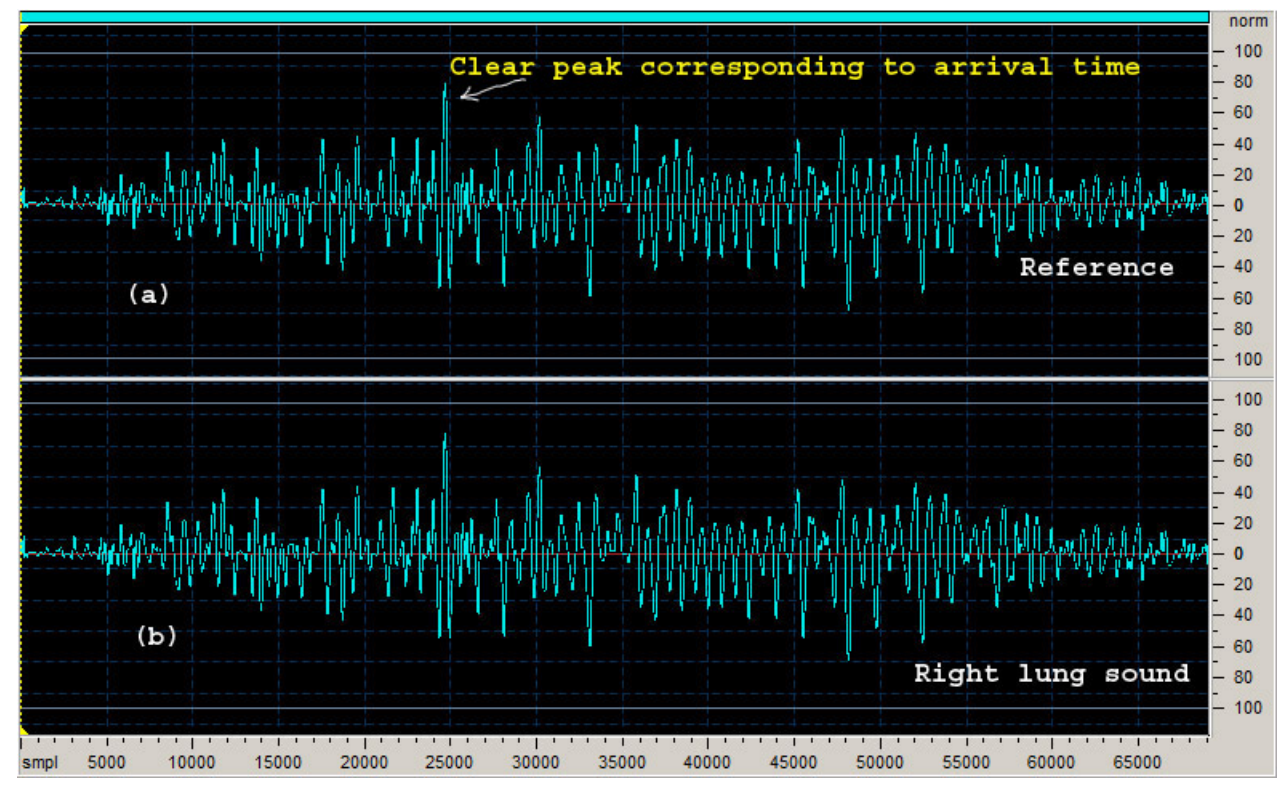

Figure 16. The transit time analysis of heart and lung sounds.

Figure 16(b), the transit time analysis was refined by cross-correlating the two sounds. The cross correlation shows a clear peak (arrow) corresponding to an arrival time difference of 14.9 ms between the difference and bottom line. Thus the correlation coefficient at the peak of the cross correlation function was 0.9 .

\section{Conclusion}

We assessed the performance of three air-coupled and four contact sensors under standardized conditions of lung sound recording. Recordings were obtained from three of the investigators at the best site on the posterior lower chest as determined by auscultation. Lung sounds were band-pass filtered between 100 and $2,000 \mathrm{~Hz}$ and sampled simultaneously with calibrated airflow at a rate of 10 $\mathrm{kHz}$. Fourier techniques were used for power spectral analysis. Average spectra for inspiratory sounds at flows of $2+/-0.5 \mathrm{~L} / \mathrm{s}$ were referenced against background noise at zero flow. Air-coupled and contact sensors had comparable maximum signal-to-noise ratios and gave similar values for most spectral parameters. Unexpectedly, less sensitivity (lower signal- to-noise ratio) at high frequencies was observed in the air-coupled devices. Sensor performance needs to be characterized in studies of lung sounds. We suggest that lung sound spectra should be averaged at known airflows over several breaths and that all measurements should be reported relative to sounds recorded at zero flow. We found that we can detect inspiratory crackle frequency had a tendency to be greater than expiratory 
frequency. While there are no immediate clinical benefits to knowing the polarity of patient's crackles, a clearer understanding of the mechanism of this prototype of aural vital-signs sounds offers the promise of improving noninvasive diagnosis of heart and lung disorders.

\section{Acknowledgements}

This work was supported by the Thailand Research Fun (TRF). The authors thank all the partners and the participants in the Electroceramic Research Unit and Applied Computer for Biomedical Engineering Unit for their help. The research has been partially supported by Professor Somkiat Wattanasirikul MD.,

\section{References}

1. Saito, N. Local Feature Extraction and Its Applications Using a Library of Bases; PhD thesis, Yale University, 1994.

2. Shino, H.; Yoshida, H.; Yana, K.; Harada, K.; Suto, J.; Harasawa, E. Detection and classification of systolic murmur for phonocardiogram; Proc. $18^{\text {th }}$ Ann.Int. ConJ IEEE EMBS, paper 1.3.1-4 on CDROM, 1996.

3. Khadra, L.; Matalgah, M.; el Asir, B.; Mawagdeh, S. The wavelet transform and its applications to phonocardiogram signal analyzing. Medical Informatics 1991, 16, 271-277.

4. Obaidat, M.S. Phonocardiogram signal analysis: techniques and performance comparison. J. Med. Eng. Technol. 1993, 17, 221-227.

5. Rumellhart, D.E.; McClelland, J.L. Purullel Distributed Processing; Vo 1.1, The MIT Press, 1986.

6. Toshitami, S. Cardiac Auscultations (In Jame); Nankodo Co., Ltd., 1994.

7. Moussavi, Z. K.; Flores, D.; Thomas, G. Heart sound cancellation based on multiscale products and linear prediction; In Proc. 26th Annu. Int. Conf. IEEE Engineering Medicine Biology Society, San Francisco, CA, 2004, 3840-3843.

(C) 2007 by MDPI (http://www.mdpi.org). Reproduction is permitted for noncommercial purposes. 